Service social

\title{
L’identité communautaire. Une perspective théorique
}

\section{Jacques Caillouette}

Volume 46, numéro 1, 1997

Politique et intervention

URI : https://id.erudit.org/iderudit/706751ar

DOI : https://doi.org/10.7202/706751ar

Aller au sommaire du numéro

Éditeur(s)

École de service social de l'Université Laval

ISSN

1708-1734 (numérique)

Découvrir la revue

Citer cet article

Caillouette, J. (1997). L’identité communautaire. Une perspective théorique. Service social, 46(1), 95-118. https://doi.org/10.7202/706751ar

\section{Résumé de l'article}

Le présent article, théorique, aborde la question de la mise en forme de l'identité communautaire. Le constructivisme sert de cadre pour penser cette identité, laquelle se pose comme constitutivede l'acteur, du mouvement social. Ce paradigme valorise la structure symbolique de l'acteur et donc la dimension culturelle des mouvements sociaux. En outre, il autorise à penser l'identité au sein de l'altérité. Les pratiques d'articulation servent à synthétiser, dans un rapport hégémonique, des positions d'acteurs différents. De cette synthèse émerge un acteur plus global en même temps que le savoir autre qui le fonde symboliquement. Une praxis cognitive marque en fait le travail de cet acteur sur le social. 


\section{L'identité communautaire Une perspective théorique}

Jacques CAILLOUETTE

Le présent article, théorique, aborde la question de la mise en forme de l'identité communautaire. Le constructivisme sert de cadre pour penser cette identité, laquelle se pose comme constitutive de l'acteur, du mouvement social. Ce paradigme valorise la structure symbolique de l'acteur et donc la dimension culturelle des mouvements sociaux. En outre, il autorise à penser l'identité au sein de l'altérité. Les pratiques d'articulation servent à synthétiser, dans un rapport hégémonique, des positions d'acteurs différents. De cette synthèse émerge un acteur plus global en même temps que le savoir autre qui le fonde symboliquement. Une praxis cognitive marque en fait le travail de cet acteur sur le social.

This article, theoretical in nature, treats the question of the shaping of community identity. Constructivism serves as the framework in conceptualizing this identity which enters into the constitution of the actor, of social movement. This paradigm focuses attention on the symbolic structure of the actor and therefore on the cultural dimension of social movements. Furthermore, it allows the placement of identity in the context of alterity. Articulatory practices serve to synthesize, in hegemonic relation, the positions of different actors. Out of this synthesis emerges a more global actor and alternative knowledge on which this actor is symbolicaly constructed. A cognitive praxis in fact marks the work of this actor in society. 


\section{INTRODUCTION}

Le but de ce texte ${ }^{1}$ est de poser la problématique de l'identité du mouvement communautaire. Andrée Fortin, dans la conclusion d'un article sur le milieu communautaire, s'interroge sur la solidarité entre les groupes communautaires et pose la question suivante: "Les coalitions qui se forment autour de certains dossiers sont-elles l'indice [...] de l'existence d'identités collectives transcendant les différents groupes? »(1991, p. 244). C'est sur cette question que je veux réfléchir, notamment en considérant la diversité identitaire que recouvre la réalité du phénomène communautaire.

Trois temps marqueront ma réflexion. II y aura d'abord le constat d'une prolifération identitaire au sein du milieu communautaire. Ensuite, j'explorerai le développement d'une perspective théorique constructiviste pour penser l'identité et le mouvement social. Dans un troisième temps, en réponse au constat de départ, j'introduirai le concept d'hégémonie élaboré dans une perspective de démocratie radicale par Ernesto Laclau et Chantal Mouffe.

\section{PLURALITÉ IDENTITAIRE DANS LE MILIEU COMMUNAUTAIRE}

On peut parler d'un phénomène de pluralité identitaire au sein du milieu communautaire. Trois points peuvent ici aider à dresser ce constat. Le premier renvoie à un article de Gérald Doré (1992) dans lequel, me semble-t-il, l'auteur pose bien le problème. Le deuxième concerne la réflexion sur l'identité qu'obligent le développement de l'économie solidaire et l'idée d'un tiers secteur communautaire pouvant lui être rattachée. Enfin, le troisième point soulevé a trait à la mobilisation large du milieu communautaire que provoque la mise en place d'un Secrétariat à l'action communautaire autonome (SACA). Ce contexte institutionnel oblige à des "investissements autoréflexifs » (Melucci, 1991) considérables de la part du milieu communautaire afin de se définir.

1. Ce texte est une version remaniée d'un exposé livré à la journée du doctorat, en novembre 1995, de l'École de service social de l'Université Laval. Cette journée s'intitulait " La complexité du communautaire ». 
Doré (1992), dans un article traitant « de la marge et du mouvement comme lieux de l'identité », fait ressortir la polarisation identitaire au sein du milieu communautaire et, conséquemment, souligne le défi que soulève cette diversité :

Au cours des années 80 , la polarisation identitaire des groupes communautaires par champ de pratique s'est accentuée. Aux regroupements qui marquent la continuité avec la décennie précédente s'en sont ajoutés de nouveaux dans une grande diversité de références identitaires: Regroupement des centres de femmes du Québec, Regroupement provincial des maisons d'hébergement et de transition pour femmes violentées, Regroupement de ressources alternatives en santé mentale du Québec, Regroupement des organismes provinciaux de personnes handicapées, Regroupement des maisons de jeunes du Québec (RMJQ), etc. (p. 146).

L'auteur fait un bilan des traits saillants de la période 19811990 et constate :

[...] malgré l'équivoque créée par la conception étatique de l'«approche communautaire » et l'émergence d'un "néo-bénévolat », un progrès quantitatif et une certaine persévérance de l'identité sont observables en organisation communautaire. II s'agit toutefois d'une identité multipolaire dont on peut appréhender qu'aucun des pôles ne soit en mesure de s'imposer comme catalyseur de l'ensemble face aux défis introduits par la nouvelle réforme des services sociaux (p. 148)

La polarisation identitaire peut laisser douter de la capacité du milieu communautaire de se trouver une composante assez forte pour articuler entre elles ses diverses parties. Or, le mouvement social demande cette articulation. Robert Mayer, Henri Lamoureux et Jean Panet-Raymond, eux aussi conscients de "l'image très contrastée » du mouvement communautaire, estiment néanmoins que le rassemblement des diverses initiatives communautaires "en un mouvement de changement social demeure un enjeu majeur pour les années à venir » (1996, p. 53). Des pratiques d'articulation, comprises en dehors de la puissance hégémonique d'une tendance particulière, représentent, à mon avis, une voie de sortie dans ce qui apparaît d'emblée comme une impasse. Mais avant d'aborder cette perspective

2. C'est moi qui souligne. 
des pratiques d'articulation, poussons plus loin la réflexion sur le thème de la prolifération identitaire.

Depuis 1990, la tendance à l'ajout de nouveaux référents identitaires s'accroît plutôt qu'elle ne se résorbe. Je pense notamment à toute la question de l'économie solidaire que plusieurs auteurs situent dans le giron communautaire (Favreau et Ninacs, 1993; Favreau, 1995; Lévesque, Joyal et Chouinard, 1989; Vaillancourt, 1996).

Par ailleurs, sur le plan théorique, certains auteurs fondent des espoirs de changement social sur la consolidation d'un secteur associatif large capable de se faire entendre politiquement au regard des grandes questions sociales. Jean Cohen et Andrew Arato (1992) parlent du secteur associatif en tant qu'expression de la société civile. S'autolimitant dans son utopie, cet acteur, sorti de sa marginalité politique, ne viserait pourtant pas à abolir le marché ou l'État. En face du marché et de l'État, il viserait plutôt à introduire une nouvelle logique dans le système, différente de la logique financière et technocrate. C'est dans cette ligne également que Jean-Louis Laville (1994) pense que les crises de l'État-providence et de la société salariale ne peuvent être résolues que par « un rapport transformé entre, d'une part, les espaces publics autonomes et, de l'autre, les sphères d'action régulées à travers l'argent et le pouvoir administratif » (p. 75, citant Habermas, 1990, p. 158).

Le Britannique Perri 6 (1994) parle d'un tiers secteur associatif en consolidation comme résultante de la jonction de deux mouvements contestataires - ou du moins qui le furent dans leur histoire - de la mise en forme capitaliste de la société : le mouvement associatif et le mouvement coopératif.

L'économiste américain Jeremy Rifkin (1995) pense pour sa part que le tiers secteur associatif permettrait d'amoindrir les effets de la période de transition et de déstructuration qu'engendrent la présente révolution informationnelle et la crise de l'emploi qui lui est liée. Plus que cela, ce secteur pourrait même jeter les bases d'une société post-marchande.

Néanmoins, comme je l'ai faitvaloir, l'hétérogénéité caractérise le «secteur communautaire ». Les contenus d'intervention, les sensibilités idéologiques, les représentations du social et les préférences pour certains types d'action varient considérablement. 
François Aubry et Jean Charest du service de recherche de la CSN ont publié un document sur l'économie solidaire. S'appuyant en partie sur des écrits de Benoît Lévesque (1994), ces deux auteurs se livrent à une énumération « plus exhaustive que limitative » des organismes qui demandent une participation directe des citoyennes et des citoyens. Cette énumération témoigne éloquemment de la diversité que recouvre le terme «communautaire ». II existe au Québec un millier d'organismes volontaires d'éducation populaire, un millier d'organismes communautaires dans le domaine de la santé et des services sociaux, plus de 600 garderies sans but lucratif, une centaine de maisons de jeunes, une centaine de centres de femmes (centres de santé, centres d'accueil, centres d'hébergement), une centaine de médias communautaires (journaux, radios, télévisions), une soixantaine de comptoirs alimentaires et de coopératives d'alimentation naturelle, de nombreuses entreprises d'insertion sociale orientées vers les services à la communauté. II existe également des organismes dans les secteurs de l'habitation (groupes de ressources techniques, OSBL d'habitation, coopératives d'habitation locative, etc.), de l'environnement, des coopératives (de production et de consommation), du développement local (coopératives de développement régional, corporations de développement économique communautaire, certains fonds régionaux et locaux d'investissement), de l'employabilité et de la recherche d'emplois. Nommons enfin les organismes de défense des droits sociaux (accidentés du travail, chômeuses et chômeurs, personnes assistées sociales, etc.), les organismes de bénévolat, les cuisines collectives, les services aux communautés culturelles (Aubry et Charest, 1995, p. 23-25).

On peut certes se demander si tous ces organismes participent vraiment d'une conscience communautaire telle qu'elle s'est développée historiquement au Québec. Toutefois, je tiens plutôt ici à diriger la réflexion sur l'articulation d'identités collectives plus larges axées sur la participation des gens à la base, des identités capables de s'imposer contre les processus d'exclusion qui empêchent une partie importante de la population de participer pleinement à l'orientation du développement sociétal. Retenons de l'énumération de Aubry et Charest que la notion de communautaire, englobant le secteur coopératif, recouvre une dispersion identitaire.

Le projet d'un Secrétariat à l'action communautaire autonome (SACA), en suscitant chez les organismes d'action communautaire 
un besoin de se définir dans leur diversité, vient lui aussi poser avec acuité la question de l'identité du mouvement social se construisant au sein même d'une diversité identitaire. Dans une proposition à ses membres, la partie communautaire du SACA écrivait que les « organismes d'action communautaire autonomes se définissent dans leur variété comme constituants d'un mouvement social autonome d'intérêt public » $(1996, \text { p. } 5)^{3}$. Or, la variété dont il est fait mention ici est large. Énumérons dix-huit des secteurs communautaires qui se mobilisent pour se définir par rapport au projet d'un SACA.

1. Le Mouvement d'éducation populaire et d'action communautaire du Québec (MÉPACQ).

2. Le Regroupement des groupes populaires d'alphabétisation du Québec (RGPAQ).

3. La Table nationale des Corporations de développement communautaires (TNCDC).

4. La Table des fédérations et des organismes nationaux d'éducation populaire autonome (ÉPA).

5. La Table des regroupements provinciaux d'organismes communautaires et bénévoles / Coalition des TROC.

6. Les organismes de femmes.

7. Les organismes de jeunes.

8. Les organismes familiaux.

9. Les groupes d'action bénévole.

10. Les organismes environnementaux.

11. Les groupes de défense de droits.

12. Les organismes actifs dans le secteur des communications.

13. Le Regroupement d'aide aux personnes handicapées.

14. Les organismes autochtones.

3. C'est moi qui souligne. 
15. Les organismes actifs dans le secteur des relations interculturelles.

16. Les organismes du secteur de l'habitation.

17. Les groupes d'aide aux immigrants et immigrantes.

18. Les organismes de loisirs.

(Boulet, 1996)

Dans un processus démocratique, ces différents types d'organismes communautaires se voient donc obligés d'arrimer leurs identités particulières afin de se définir en commun et, du même coup, de redéfinir le projet gouvernemental d'un SACA de façon que ce dernier respecte l'identité de l'action communautaire. Cet exemple montre lui aussi que l'identité multipolaire du mouvement communautaire québécois constitue un défi pour la consolidation et le développement de ce mouvement.

En fait, que ce soit devant les différents types d'acteurs se mobilisant pour redéfinir le projet d'un SACA, ou devant la perspective de l'économie solidaire et d'un tiers secteur, ou encore devant la réalité d'une identité multipolaire au sein du milieu communautaire, on est en droit de se demander si, au cœur de cette prolifération identitaire, l'articulation de cet ensemble hétérogène autour d'un lien identitaire commun est possible.

Pour répondre à cette question, il est opportun maintenant d'aborder les concepts d'identité, d'acteur social et de pratiques d'articulation en faisant référence aux théories sur les mouvements sociaux.

\section{LA QUESTION IDENTITAIRE : UNE PERSPECTIVE THÉORIQUE}

\section{Le mouvement social comme acteur et sujet}

Le concept d'identité se rattache à ceux d'acteur social et de sujet social. Le processus identitaire, dans l'optique des mouvements sociaux, renvoie, pour sa part, aux modes de création et de formation de l'acteur qui ne peut se poser comme tel qu'en raison de sa conscience d'acteur (Touraine, 1978, p. 93). Or, cette conscience se construit dans une lutte contre l'aliénation et participe à un processus de subjectivation au sens d'une affirmation du sujet collectif. Ce dernier se construit et se définit par sa lutte contre les forces sociales qui posent comme objet les membres de la société. C'est en ce sens que Touraine (1992, p. 401) affirme que les processus de subjectivation se fondent 
sur des valeurs de liberté, d'indépendance et de responsabilité et que " le mouvement social est l'action collective de défense du sujet contre le pouvoir de la marchandise, de l'entreprise et de l'État » (1992, p. 331). Pour le présent exposé où il est question de diversité communautaire, un autre point à retenir de la pensée de Touraine est que l'acteur, dans sa construction identitaire, dans son processus de subjectivation, évite de nier l'autre dans sa qualité de sujet: "le sujet se construit à la fois par la lutte contre les appareils et par le respect de l'autre comme sujet »(1992, p. 331). La construction identitaire se comprend donc comme le passage, pour des individus ou des groupes sociaux, d'un statut d'objet à celui de sujet, et ce processus de subjectivation se bâtit dans des espaces communicationnels ouverts à l'altérité.

Ayant à l'esprit le milieu communautaire québécois, engagé de plus en plus dans une dynamique partenariale avec l'État, j'appellerai acteur celui qui, partant d'une logique communautaire enracinée dans le monde vécu, se constitue, dans un esprit démocratique, comme sujet soucieux de participer aux champs politique, social, culturel et économique afin d'influencer l'orientation sociétale, de dévoiler le présent ordre des choses et d'en négocier un nouveau. L'acteur est donc porteur d'une transformation sociale. Objets d'une situation sociale, les individus, groupes ou populations se constituent en acteurs lorsque, grâce à leur mobilisation, ils réussissent à se définir de manière à remettre en question, à défier et à redéfinir selon leur propre identité les réponses, ou les non-réponses, apportées à leur situation par des autorités, des appareils ou des mécanismes déniant leur qualité de sujets. L'identité, quant à elle, est fondatrice de l'acteur.

\section{L'identité comme construit et discours}

Le constructivisme remet en question la "positivité » du social. Peter Berger et Thomas Luckmann (1979) parleront en ce sens de la "construction sociale de la réalité ». Ce qui est dénié n'est pas que les objets existent à l'extérieur de la pensée, mais bien que ces objets ne pourraient point eux-mêmes se conceptualiser comme tels à l'extérieur d'un processus de cognition. La division subjectif/objectif s'effondre. Le subjectif et l'objectif, indissociables, constituent le social (Laclau et Mouffe, 1993, p. 108 et 
110). Le symbolique, en tant que réflexivité de l'action, s'incorpore entièrement à la réalité " objective » où il prend forme tout en donnant forme.

C'est lorsqu'on la compare à une perspective essentialiste de l'identité que l'approche constructiviste prend toute sa couleur. Appliqué à la réalité communautaire, un discours essentialiste présupposerait une identité communautaire objective qu'il s'agirait d'actualiser sur le plan subjectif. La représentation subjective de l'acteur, pour qu'elle soit vraie, devrait alors refléter les conditions objectives de son existence économique et sociale. Dans la perspective essentialiste, l'acteur se doit de "découvrir » - et non de construire et d'interpréter - son identité. Celle-ci le précède. Elle existe avant même qu'il ait pu s'en saisir. II s'agit dès lors pour l'agent de l'action historique de coller subjectivement à sa nature, à sa mission et à son destin, lesquels s'inscrivent de manière univoque dans l'ordre social, historique ou économique.

Dans la perspective constructiviste, au contraire, la réalité " objective » n'existe pas en soi. Elle passe obligatoirement par un processus de cognition. L'identité devient alors un construit cognitif, une connaissance à partir d'un champ social donné. Et cette connaissance, en contribuant à la construction du champ social où se mobilisent les acteurs, s'insère dans ce champ comme un élément lui étant constitutif. Le discours sur soi et sur son environnement, dans la mesure où il devient prégnant socialement, est constitutif de l'acteur. Ce dernier devient à la fois cause et effet de sa représentation du réel. Plus que le représentant d'intérêts particuliers, il est, dans cette perspective, le tenant d'un autre savoir 4 .

Le discours du mouvement social renvoie à l'expression d'une connaissance particulière qui, dans sa constitution même, se veut action transformatrice de ce réel. Un peu comme «les scientifiques produisent les représentations du monde qui peuvent être utiles en vue d'une maîtrise matérielle de celui-ci » (Fourez, 1988, p. 102), le mouvement social, dans son action et

4. Jean Lecuit (1993) développe ce thème d'un "autre savoir » en l'appliquant à la connaissance que recèle le contenu des histoires de vie que les membres du mouvement quart-monde réalisent avec des personnes vivant une exclusion sociale extrême. Sur le plan de la sociologie de la connaissance, la perspective de cet auteur peut être utile pour mieux comprendre la nature des mouvements sociaux. 
pour elle, produit des représentations utiles au changement social et dans ce processus se produit lui-même comme mouvement.

L'affirmation identitaire prise comme expression et défense par l'acteur de sa vision du monde constitue son action sur ce monde. L'acteur se forme au sein même de son expression. C'est en ce sens que le processus identitaire fonde le mouvement social. Car le sujet, le mouvement social, n'est pas d'abord la rencontre d'individus ou l'interaction de ces individus qui nouent des liens entre eux; il se réalise essentiellement lorsque les gens se créent ensemble et partagent une identité autour d'une expression commune. Comme le dit Emmanuel Lévinas, dans un autre contexte, "les personnes ne sont pas l'une devant l'autre, simplement elles sont les unes avec les autres autour de quelque chose » (1981, p. 62).

Mais ce quelque chose, ce sont les personnes et les groupes qui le font émerger. Par leur agir communicationnel et en partant d'eux-mêmes, ils réussissent à se saisir et à se vivre dans une identité particulière. Le discours identitaire est en fait tributaire de la rencontre d'une histoire factuelle, nécessairement équivoque, et de différentes identités en interaction qui, participant à cette histoire, l'énoncent en lui conférant un sens particulier.

C'est par cette recherche de sens que l'identité s'articule et que l'acteur, porteur d'un autre savoir, se constitue. Mais, pour que ce savoir soit autre, il faut que celles et ceux qui l'énoncent le construisent à partir d'eux-mêmes. La constitution de ce savoir demande un acte de liberté. Toute aliénation l'entrave. Comme Touraine (1992) le fait si bien valoir, l'acteur ne peut pas découler d'une logique systémique, il est en lutte contre toute réification de son être.

C'est dans cette perspective qu'il convient d'aborder l'action communautaire comme porteuse de changement social. Travail en faveur des exclus, l'action communautaire est transformatrice lorsqu'elle participe avec eux à la promotion de manières de faire, de voir et de connaître capables d'aider la société dans son ensemble à se voir autrement et à agir d'une autre manière sur elle-même. Même si la " praxis cognitive » (Eyerman et Jamison, 1991) part d'acteurs positionnés dans l'espace social et est donc productrice d'une connaissance intéressée du social, elle ne se réduit pas à une défense d'intérêts, car elle est volonté de 
participer à la définition du social dans son ensemble. L'identité découlant de la praxis se relie en fait au concept d'historicité qui présuppose que les sociétés modernes sont capables de produire leurs orientations culturelles et sociales en vue d'une action transformatrice sur elles-mêmes (Touraine, 1973, p. 33 et 1992, p. 423). Le mouvement social se relie à l'historicité, il est volonté d'action sur elle. Les identités nouvelles qu'impliquent les savoirs alternatifs dépassent la défense d'intérêts; elles remettent en question la définition du jeu des différents acteurs sociaux et de leurs identités respectives.

L'appartenance identitaire, même si elle peut s'y appuyer, ne se fonde pas nécessairement sur l'intérêt à être ensemble, sur le calcul stratégique de la part des individus ou des groupes pour adhérer au mouvement. L'appartenance identitaire est la participation à un projet culturel commun: "le problème de la représentation se pose plus en termes cognitifs qu'en termes identitaires d'intérêt » dira Pierre Rosanvallon (1995, p. 41). La construction identitaire revient donc à la production en commun d'une structure cognitive d'appréhension du social et de son devenir. Elle est création interactive d'un imaginaire partagé qui s'exprime dans des manières d'être, de faire et de voir. Loin de se limiter à la défense d'intérêts, l'identité est culture. Elle résulte beaucoup plus d'une production en commun que du partage d'une vision déjà là à laquelle il faudrait se plier. La lutte de l'acteur, celle de son autonomie, doit s'observer non pas tant sous l'angle du calcul stratégique à visée corporatiste que sous celui d'une perspective culturelle et politique où, à partir de sa base, le mouvement se construit dans une action de modification des orientations sociétales.

Cette perspective constructiviste de l'identité autorise une analyse beaucoup plus politique qu'économique des conflits sociaux (Melucci, 1995, p. 201) et donc de la création des acteurs. La créativité, la volonté politique et le débat démocratique prennent l'avant-scène des processus de mise en forme identitaire.

\section{L'identité comme histoire}

Quoique l'étude des mouvements sociaux, axée ici sur la question de l'identité, mette fortement en relief la créativité symbolique des acteurs, il ne faudrait pas pour autant laisser croire que l'identité procède d'un volontarisme arbitraire. La perspective constructiviste n'accorde pas une autonomie sans bornes aux 
représentations des acteurs. Les représentations se rattachent à des réalités existentielles. Plus qu'un habillement, les représentations sociales sont ce par quoi les acteurs existent socialement. Ces représentations ne peuvent donc pas se métamorphoser sous la simple impulsion d'une volonté conquérante. Le sens nouveau qui émerge, la connaissance nouvelle de soi, la réinterprétation de son histoire ont toujours besoin d'un substrat, d'une réalité factuelle et reconnue, auxquels s'intégrer. Les ruptures se produisent, mais dans le cadre de renouvellements et de continuités identitaires. En réalité, il faudrait dire que les porteurs de l'identité la saisissent créativement beaucoup plus qu'ils ne la produisent. Des identités antérieures précèdent celles en formation. Bref, si l'identité est innovation, elle est aussi mémoire, mais une mémoire vivante! C'est que l'identité, pour se construire, part d'un vécu et d'un patrimoine symbolique déjà existants qui d'un côté s'imposent à elle, mais de l'autre demeurent toujours sujets à une réinterprétation.

Contre un volontarisme des représentations, notons de plus que ces dernières se construisent dans un contexte sociohistorique, politique et culturel particulier marqué par des rapports d'inégalité et de domination qui s'incarnent dans des institutions et des organisations. Bref, la perspective défendue ici ne prétend pas qu'on puisse affirmer n'importe quoi sur le plan identitaire; elle insiste tout simplement sur le fait que le processus identitaire en tant qu'activité symbolique laisse un large espace à l'action et à la réflexion de celles et de ceux qui se rencontrent dans cette identité pour l'énoncer et la partager.

\section{L'identité comme espace}

La constitution de l'action et la constitution de l'acteur se répondent mutuellement. L'identité informe la pratique en même temps qu'elle est conscience réflexive de cette pratique. L'identité informe la pratique, car l'action provient de l'acteur et celui-ci porte une conscience particulière de lui-même qui conditionne amplement la mise en forme de son action. Hamel souligne que :

[...] la marge de manœuvre dont disposent les acteurs d'un mouvement ne résulte pas uniquement des contraintes qui proviennent de l'environnement mais aussi des possibilités et des limites qui découlent du "schéma général » 
qu'ils ont adopté au départ $(1995$, p. 286 , se référant à Snow et Bedford, 1992, p. 146).

La représentation de soi, c'est-à-dire la manière dont l'acteur se conçoit et se crée, et la présentation de soi qui lui importe étant donné son identité viennent informer son action, de même que les moyens qu'il va utiliser pour construire cette action. Mais, à l'inverse, si l'identité informe l'action, elle est également construction et saisie de soi dans l'action. L'acteur naît de son action autant qu'il la façonne. Le processus identitaire qui le fonde comme acteur est aussi réflexivité sur sa pratique. Plus que des agents se limitant à répondre à une situation donnée, les personnes ou les groupes investissent symboliquement leur situation et leur action pour y introduire du sens. Dans cet univers significatif, ils se donnent symboliquement un lieu, un être, à partir desquels ils peuvent agir, se comprendre dans leur action et informer les autres du sens de celle-ci.

Bien entendu, tout ce travail identitaire se déploie dans un espace sociohistorique qui ouvre sur des possibles, mais oblige aussi à des contraintes. II ne s'agit pas à vrai dire de produire le sens et l'identité, mais de les faire advenir au sein d'événements que l'on crée, qui nous arrivent et auxquels on participe. Fernand Dumont (1968) parle de la culture comme du lieu de I'homme, de l'humain. La construction identitaire peut elle aussi se comprendre comme l'aménagement d'un lieu symbolique qui permet la création de l'acteur et de son action.

Lorsqu'on parle de l'identité, il s'agit donc de la construction d'un sujet et aussi d'un espace où peut se développer ce sujet. II y a un travail sur soi à faire et un travail politique à accomplir afin de libérer l'espace nécessaire à l'affirmation de soi et au déploiement de son activité comme sujet. L'un va avec l'autre. La construction de l'acteur, si elle exige un travail de définition de soi, de mise en relation, de synthèse identitaire, est de surcroît un combat pour les moyens et les ressources nécessaires à l'affirmation et à la consolidation identitaires.

L'identité a besoin d'un lieu d'énonciation. Elle résulte d'une créativité symbolique soutenue par une habileté organisationnelle et relationnelle de personnes ou de groupes qui se rencontrent dans un espace de désirs, de contraintes et d'opportunités, et qui réussissent à se poser, à se construire et à se reconnaître ensemble comme des sujets prenant prise sur I'histoire. 
Le discours identitaire, avons-nous fait valoir, n'est pas l'expression d'un acteur qui pourrait exister "objectivement " à l'extérieur de son discours. Le mouvement social se forme dans l'énonciation même de son discours, qui lui est constitutif (Laclau et Mouffe, 1993). En refusant des déterminants métasociaux, déistes ou naturistes pour expliquer le social, l'acteur se libère un espace communicationnel d'intersubjectivité et d'échange démocratiques, mais il lui faut également conquérir cet espace sur les plans organisationnel et institutionnel. Eyerman et Jamison insistent sur cet aspect. Ils font valoir que, pour le mouvement social, "le sens, l'identité centrale, est l'espace cognitif que le mouvement crée, un espace où de nouvelles sortes d'idées et de relations peuvent se développer » $(1991, \text { p. 60 })^{5}$.

Cette perspective pour saisir l'identité redonne toute son importance à l'activité symbolique. Pour la recherche comme pour la pratique, elle invite à porter une attention sur les systèmes de signes et de sens en émergence et à relier ceux-ci à leur potentiel d'action. C'est dans cet univers sémiotique que Melucci conçoit l'élaboration identitaire en tant que processus de « construction » d'un système d'action. Plus précisément,

[...] l'identité collective est, selon lui, une définition interactive et partagée produite par quelques individus (ou groupes à un niveau plus complexe) et portant sur les orientations de l'action, laquelle s'inscrit dans un champ d'opportunités et de contraintes. Par « interactif et partagé », j'entends, ajoute-t-il, une définition qui doit être prise comme un processus étant donné que cette définition est construite et négociée au travers d'une activation répétée du réseau relationnel qui lie les individus (ou les groupes) porteurs de cette définition (1995, p. 44).

\section{La construction identitaire comme action sur le social}

Si l'identité relève d'une négociation interne, elle doit également composer avec un milieu externe pouvant être à certains égards hostile au développement du nouvel acteur. C'est dans la saisie de soi, dans l'autoconscience que se crée l'acteur, c'est en quelque sorte le «je pense donc je suis » de Descartes. Mais

5. Dans ce texte, les citations tirées d'ouvrages anglophones et traduites en français le sont par l'auteur. 
cette saisie particulière de soi, ce nouveau savoir mis en action affronte d'autres représentations du social et du politique. L'acteur se construit bien souvent au sein de rapports sociaux d'inégalité et de domination qui justement lui nient l'autonomie de se définir et de se poser comme acteur du changement social.

Pierre Bourdieu conçoit les luttes pour la définition des identités ethnique ou régionale comme des « luttes pour le monopole du pouvoir de faire voir et de faire croire, de faire connaître et de faire reconnaître, d'imposer la définition légitime des divisions du monde social et, par là, de faire et de défaire les groupes (1982, p. 137$)^{6}$. En se construisant, c'est dans cet univers qu'intervient le mouvement social. Par exemple, le féminisme est un savoir qui, en fait, est la conscience du mouvement des femmes. Le féminisme donne corps à ce mouvement, et c'est l'amplitude de ce mouvement qui en fait un savoir plus ou moins prégnant socialement. Or, le féminisme, ce savoir particulier, est la raison d'être du mouvement des femmes. Ce mouvement, dans la mesure où il réussit à imprimer sa pensée dans le social, devient un acteur du changement puisqu'il défend une autre vision des rapports hommes-femmes que celle instituée.

Eyerman et Jamison (1991) soulignent comment la construction symbolique de l'acteur est à la fois condition en même temps que but de l'action de transformation sociale:

Ce que nous défendons est que les mouvements sociaux élaborent de nouveaux projets historiques en construisant sur le plan cognitif leur propre identité. En formulant ce qu'ils assument comme étant la réalité sociale, en développant la présentation "programmatique " d'eux-mêmes au reste de la société; bref, en nommant la cause pour laquelle ils se tiennent debout, les activistes des mouvements sociaux développent de nouvelles idées qui se situent au cœur des processus plus larges de la créativité humaine (p. 165).

Comme pour le mouvement féministe, on peut dire que l'émergence d'une conscience communautaire autonome représente le défi du milieu communautaire lorsqu'il tend vers le mouvement social. C'est dans la mesure où une conscience particulière, portée par un mouvement et porteuse de ce mouvement, s'implante dans le social qu'elle peut être transformatrice sur les plans culturel, politique et économique.

6. C'est Bourdieu qui souligne. 
Toutefois, il faut comprendre que le développement de cette conscience est un enjeu social et politique. Par exemple, c'est un défi pour le milieu communautaire de se poser comme sujet visà-vis des tendances marchande et technocratique qui, poids lourds de la modernité, tendent à soumettre à une participation dépendante les systèmes d'action qui leur sont étrangers. Dès lors, on doit certes s'attendre à repérer dans le milieu communautaire des volontés collectives dirigées vers une affirmation d'autonomie, d'initiative et de responsabilité, et en même temps à les concevoir en conflit avec des forces d'imposition identitaire. Dans les sociétés industrielles avancées, les grands appareils de pouvoir tendent à modeler les conduites sociales et culturelles. Maheu dira par exemple que l'imposition et l'attribution autoritaire d'identité s'appuient "sur des agences et des mécanismes d'imposition comme le marché de la consommation », et sur " les appareils de prise en charge, d'aide et de soutien du domaine de la santé et du bien-être » (1991, p. 179). Des forces externes au mouvement peuvent donc tenter de le soumettre à une participation dépendante. Cependant, ces forces adverses constituent pour le mouvement une occasion de s'autoconscientiser comme porteur d'une différence. En fait, l'identité semble se créer au sein même de sa négation : "Imposition autoritaire d'identité et processus sous-tendant l'autonomisation des sujets sont en fréquentes tensions dialectiques dans nombre de systèmes d'action et de conflits sociaux » (p. 187).

Pour le communautaire, l'identité semble se jouer entre deux grands pôles. Soit le milieu communautaire se fait étatiser et aliène son être au profit d'une rationalité technocratique, voire marchande, qui l'instrumentalise, soit, profitant de l'appel au partenariat qu'implique une nouvelle gestion du social (Lamoureux, 1994), il réussit à se poser en partenaire autonome, à obtenir un plus grand accès à diverses ressources et, de cette manière, à soutenir plus adéquatement au sein de la société civile l'émergence et la consolidation de dynamiques communautaires autonomes. Autrement dit, l'affirmation identitaire d'un côté se pose comme un contrepoids aux régulations technicienne et marchande, et de l'autre comme une volonté de renforcer le pouvoir d'action des personnes et des groupes marginalisés afin qu'ils puissent participer pleinement, et selon leur culture propre, au développement sociétal et à son orientation. 
La construction cognitive qu'implique l'identité ne s'accomplit donc pas dans un vide social. Selon Touraine (1995) :

[...] le sujet devient visible et autoconscient à travers sa lutte contre les forces qui tendent à le transformer en objet. Les individus ou les groupes deviennent sujets non parce qu'ils expriment leurs idées, mais parce qu'ils se libèrent eux-mêmes; ils se dégagent des catégories, régulations ou influences qui les réduisent à des éléments de systèmes qu'ils ne contrôlent pas (p. 272).

Le processus identitaire renvoie à des négociations à l'intérieur et à l'extérieur du mouvement : à l'interne, puisqu'il faut prendre en considération les processus relationnels et d'interaction entre les diverses composantes à l'intérieur du mouvement; à l'externe, puisqu'il faut prendre en considération les relations et les interactions que ce système d'action entretient avec son environnement extérieur, dont les pouvoirs étatiques et marchands. Melucci dira en ce sens que «l'identité d'un mouvement est le produit de la négociation interne et des relations avec le milieu systémique » (1991, p. 154).

\section{PRATIQUES D'ARTICULATION ET DE CONSTRUCTION IDENTITAIRES}

Pour faire suite à la perspective plus sociologique élaborée précédemment, il convient maintenant d'aborder le processus identitaire sous un angle plus politique. Le milieu communautaire est diversifié. Comment peut-il donc développer une cohésion d'acteur au sein de cette diversité?

On peut concevoir la société québécoise au travers d'une prolifération de points de rupture. II n'est pas surprenant alors que la diversité marque l'action des divers groupes bénévoles, communautaires ou populaires. Selon le groupe, l'accent portera sur le féminisme, la lutte à l'exclusion, la défense de la citoyenneté, l'engagement bénévole, etc.

Le milieu communautaire peut se construire comme un espace d'intersubjectivité où se rencontrent diverses identités. Cet espace devient alors non pas tant le lieu des dénominateurs communs qu'un espace communicationnel autonome créé en commun. L'acteur, loin de gommer sa diversité, s'appuie sur elle pour se fonder. De son hétérogénéité reconnue, assumée et valorisée, il tire la créativité et les ressources nécessaires au renouvellement continuel de son action. Selon les contextes et 
les enjeux, l'activation et l'articulation des différents réseaux qui le composent permettent au mouvement de maintenir toujours présente une capacité de remise en question.

Ernesto Laclau et Chantal Mouffe (1993) ouvrent en ce sens des perspectives heuristiques. Dans une optique de démocratie radicale et pluraliste, ces auteurs font appel à la construction discursive et politique d'un bloc historique liant divers mouvements sociaux, sans pour autant oblitérer leurs identités respectives. Le processus identitaire se manifeste dès lors comme la rencontre d'identités particulières d'où émerge une nouvelle conscience plus large qui se répercute sur ses parties constituantes. Les pratiques d'articulation, pour Laclau et Mouffe, jouent un rôle fondamental dans le processus de construction identitaire :

[...] nous appellerons articulation toute pratique qui éta-

blit une relation parmi des éléments de telle manière que leur identité est modifiée en raison de la pratique articulatoire (p. 105)

Cette nouvelle construction identitaire résultant des pratiques d'articulation se qualifie d'hégémonique. II faut entendre le concept au sens de Gramsci. Cependant, à la différence de ce théoricien, la construction du lien hégémonique ne fait pas appel ici à la prédominance d'une identité sur les autres. Si l'on applique ce concept au milieu communautaire, on peut penser que différentes consciences, différentes éthiques (Lamoureux, 1996) à l'intérieur de la mouvance communautaire peuvent s'articuler entre elles de manière à déboucher sur de nouvelles pratiques discursives.

L'idée d'une identité hégémonique rejoint la perspective de Eyerman et Jamison pour qui le mouvement social est " un territoire cognitif, un nouvel espace conceptuel, où se vit une dynamique interactionnelle entre différents groupes et organisations ». Ces auteurs ajoutent que " c'est au travers des tensions entre les différentes organisations pour se définir et agir dans cet espace conceptuel que l'identité (temporaire) du mouvement social se forme " (Eyerman et Jamison, 1991, p. 55). Espace hégémonique et espace conceptuel réfèrent tous deux à un lieu où différents sens se fécondent mutuellement dans une recherche d'imaginaires nouveaux pour mener l'action de changement 
social. La participation au lien hégémonique est transformatrice, car la nouvelle identité plus large en construction rejaillit sur les identités particulières qui la composent. Elle leur offre un nouveau lieu pour se penser. Dans ce processus, les éléments qui se rencontrent sont modifiés du fait même de leur rencontre.

L'optique constructiviste ici postulée amène à concevoir la précarité de toute identité et l'impossibilité d'en fixer le sens une fois pour toutes. Et si une construction hégémonique se figeait sur le plan identitaire, c'est qu'entre ses diverses composantes prévaudrait une relation de subordination et de pouvoir, mais non une relation hégémonique. Le lien hégémonique, pour demeurer vivant, demande une communication toujours ouverte entre celles et ceux qui participent à son être.

Pour terminer sur les pratiques d'articulation et la perspective constructiviste de l'identité que cette notion sous-tend, recourons à la pensée de Klaus Eder (1995) sur la construction de classe. Cet auteur parle d'opportunités structurelles (social opportunity structure) pour désigner l'espace social d'où la classe peut émerger. Eder précise que la construction de la classe, l'établissement de ses frontières et du type de relations qu'elle entretiendra avec les autres classes relèvent d'un travail de mobilisation et d'action collectives des différents acteurs de cette classe en formation. C'est ainsi que pour eux « la classe est une construction sociale qui réunit ensemble des catégories sociales de manière à former un tout plus englobant [...] » (Eder, 1995, p. 41).

Eder, ayant à l'esprit les nouveaux mouvements sociaux, parle de la fabrication (the making) de la classe sociale comme résultant d'une praxis collective où les acteurs créent leur conscience de classe en même temps qu'ils se créent comme classe. Eder, Mouffe et Laclau ainsi que Eyerman et Jamison partagent l'idée que la création du mouvement social découle de pratiques d'articulation constitutives d'un lien hégémonique démocratique ou d'une dynamique interactionnelle menant à la création d'un espace conceptuel nouveau.

Ces perspectives théoriques jointes à une conception du mouvement social comme autoconstruction de l'acteur autorisent à concevoir comme possible l'émergence d'un mouvement communautaire large possédant la masse critique nécessaire pour générer des énergies capables d'apporter à la société une conception renouvelée d'elle-même. II est à espérer qu'au Québec, ces années-ci, puissent se développer de tels processus de 
renouvellement identitaire de manière à contrer la prégnance des imaginaires marchand et technocratique dominants. Lorsqu'ils sont laissés à eux-mêmes, ceux-ci tendent à repousser l'humain à leur périphérie plutôt que d'être des instruments pour son émancipation.

Jacques CAILLOUETTE

Professeur

Département de service social

Université de Sherbrooke 


\section{Références bibliographiques}

6, PERRI (1994). "Introduction » (p. 1-17), dans Perri 6 et Isabelle Vidal (dir.), Delivering Welfare. Repositioning Non-profit and Co-operative Action in Western European Welfare States, Barcelonia, CIES Centre d'Iniciatives de l'Economia Social, $409 \mathrm{p}$.

AUBRY, François et Jean CHAREST (recherche et rédaction) (1995). Développer l'économie solidaire. Éléments d'orientation, Service de la recherche CSN, octobre, $50 \mathrm{p}$.

BERGER, Peter et Thomas LUCKMANN (1979). The Social Construction of Reality. A Treatise in the Sociology of Knowledge, New York, Penguin Books, 249 p.

BOULET, Christine (1996). La saga du SACA: historique, Mouvement populaire et communautaire, 4 mai, p. 2.

BOURDIEU, Pierre (1982). Ce que parler veut dire. L'économie des échanges linguistiques, Paris, Fayard, 244 p.

CAILLOUETTE, Jacques (1992). "La réforme Côté ou l'ambivalence de l'Étatà l'égard du communautaire », Service social, vol. 41, $n^{\circ} 2$, p. $115-131$.

CAILLOUETTE, Jacques (1994). "L'État partenaire du communautaire: vers un nouveau modèle de développement ", Nouvelles pratiques sociales, vol. $7, n^{\circ} 1, p .161-175$.

COHEN, Jean L. et Andrew ARATO (1992). Civil Society and Political Theory, Cambridge, Massachusetts, MIT Press, $771 \mathrm{p}$.

DORÉ, Gérald (1992), "L'organisation communautaire et les mutations dans les services sociaux au Québec, 1961-1991. La marge et le mouvement comme lieux de l'identité ", Service social, vol. $41, n^{\circ} 2$, p. 131-162.

DUMONT, Fernand (1994). Le lieu de l'homme. Présentation de Serge Cantin, 1968 [...] Bibliothèque québécoise, $284 \mathrm{p}$.

EDER, Klaus (1995). "Does Social Class Matter in the Study of Social Movements? A Theory of Middle-class Radicalism " (p. 21-54), dans Louis Maheu (dir.), Social Movements and Social Classes. The Future of Collective Action, Thousand Oaks, CA, Sage, $280 \mathrm{p}$.

EYERMANE, Ron et Andrew JAMISON (1991). Social Movements. A Cognitive Approach, University Park, PA, The Pennsylvania State University Press, $184 \mathrm{p}$.

FAVREAU, Louis et William A. NINACS (1993). Pratiques de développement économique communautaire au Québec: de l'expérimentation sociale à l'émergence d'une économie soli- 
daire, Résumé du rapport d'une recherche financée par le Programme des Subventions nationales au bien-être social de Développement des ressources humaines Canada, novembre, $40 \mathrm{p}$.

FAVREAU, Louis (1995). Repenser le mouvement communautaire dans une perspective d'économie solidaire. Une hypothèse de renouvellement de la problématique et de la politique du développement communautaire et de l'économie sociale au Québec, Cahiers du Collectif de recherche sur les innovations sociales dans les entreprises et les syndicats (CRISES), Coll. Économie sociale, UQAM, UQAR, UQAH, Université Laval, septembre, $27 \mathrm{p}$.

FORTIN, Andrée (1991). "La participation: des comités de citoyens au mouvement communautaire » (p. 219-250), dans Jacques T. Godbout (dir.), La participation politique, Québec, Institut québécois de recherche sur la culture, $301 \mathrm{p}$.

FOUREZ, Gérard (1988). La construction des sciences. Introduction à la Philosophie et à l'Éthique des sciences, Bruxelles, De Boeck, $235 \mathrm{p}$.

HABERMAS, Jürgen (1990). Écrits politiques : culture, droit, histoire (1985), Paris, Cerf, 263 p.

HAMEL, Pierre (1995). "Mouvements urbains et modernité : l'exemple montréalais ", Recherches sociographiques, vol. XXXVI, $n^{\circ}$ 2, p. 279-305.

LACLAU, Ernesto et Chantal MOUFFE (1993). Hegemony and Socialist Strategy: Towards a Radical Democratic Politics, 1985, Londres, Verso, $197 \mathrm{p}$.

LAMOUREUX, Jocelyne (1994). Le partenariat à l'épreuve, Montréal, Éditions Saint-Martin, $235 \mathrm{p}$.

LAMOUREUX, Henri (1996). Le citoyen responsable, Montréal, VLB éditeur, $197 \mathrm{p}$.

LAVILLE, Jean-Louis (1994). "État et société au défi de la solidarité », Esprit, août-septembre, p. 69-79.

LECUIT, Jean (1993). Un autre savoir. À l'école des plus pauvres, Paris, Éditions Quart monde, $289 \mathrm{p}$.

LÉVESQUE, Benoît (1994). "Québec: des expériences à l'institutionnalisation », dans Bernard Eme et Jean-Louis Laville (dir.), Cohésion sociale et emploi, Paris, Brouwer. 
LÉVESQUE, Benoît, André JOYAL et Omer CHOUINARD (dir.) (1989). L'autre économie. Une économie alternative? SainteFoy, Presses de l'Université du Québec, 372 p.

LÉVINAS, Emmanuel (1981). De l'existence à l'existant, $2^{\mathrm{e}}$ éd. augm., Paris, Librairie philosophique J. Vrin, $174 \mathrm{p}$.

MAHEU, Louis (1991). "Les nouveaux mouvements sociaux entre les voies de l'identité et les enjeux du politique " (p. 163192), dans Louis Maheu et Arnaud Sales (dir.), La recomposition du politique, Paris et Montréal, L'Harmattan et Presses de l'Université de Montréal, 324 p.

MAYER, Robert, Henri LAMOUREUX et Jean PANET-RAYMOND (1996). "Évolution des pratiques communautaires au Québec » (p. 7-72), dans Henri Lamoureux, Jocelyne Lavoie, Robert Mayer et Jean Panet-Raymond (dir.), La pratique de l'action communautaire, Sainte-Foy, Presses de l'Université du Québec, $436 \mathrm{p}$.

MELUCCI, Alberto (1995). "The Process of Collective Identity » (p. 41-63), dans Hand Johnston et Bert Klandermans (dir.), Social Movements and Culture, Social Movements, Protest, and Contention, vol. 4, Minneapolis, MA, University of Minnesota Press, $281 \mathrm{p}$.

MELUCCI, Alberto (1991). "Qu'y a-t-il de nouveau dans les "nouveaux mouvements sociaux"? "(p. 129-162), dans Louis Maheu et Arnaud Sales (dir.), La recomposition du politique, Paris et Montréal, L'Harmattan et Presses de l'Université de Montréal, $324 \mathrm{p}$.

RIFKIN, Jeremy (1995). The End of Work, New York, GP Putnam's Sons, $350 \mathrm{p}$.

ROSANVALLON, Pierre (1995). "Faire l'histoire du politique » (p. 25-42). Entretien avec Pierre Rosanvallon, propos recueillis par Pierre Bouretz, Olivier Mangin et Joël Roman, Esprit, février, $209 \mathrm{p}$.

SACA, MEMBRES COMMUNAUTAIRES DU COMITÉ AVISEUR PROVISOIRE (1996). Pour la reconnaissance et le financement de l'action communautaire autonome, Proposition commune soumise aux organismes des secteurs membres du Comité aviseur provisoire du Secrétariat à l'action communautaire autonome (SACA), Rencontre nationale, 14 et 15 novembre 1996, $12 \mathrm{p}$.

TOURAINE, Alain (1995). "Democracy: From a Politics of Citizenship to a Politics of Recognition » (p. 258-275), dans Louis Maheu (dir.), Social Movements and Social Classes. The Future of Collective Action, Thousand Oaks, CA, Sage, 280 p. 
TOURAINE, Alain (1992). Critique de la modernité, Paris, Fayard, $462 \mathrm{p}$.

TOURAINE, Alain (1978). La voix et le regard, Paris, Seuil, 305 p.

TOURAINE, Alain (1973). Production de la société, Paris, Seuil, $543 \mathrm{p}$.

VAILLANCOURT, Yves (1996). Comment poser le problème de la privatisation dans le réseau de la santé et des services sociaux au Québec. Texte à paraître dans une publication préparée sous la direction de Jean-Louis Laville, Montréal, février, $59 \mathrm{p}$.

YAKABUSKI, Konrad (1995). "Les groupes communautaires redoutent le diktat de Lisette Lapointe », Le Devoir, p. A4. 